\title{
Updated Fermi-LAT Constraints on the Extragalactic Background Light
}

\section{Thomas Armstrong*}

Department of Astrophysics

University of Oxford

Denys Wilkinson Building

1 Keble Road, Oxford

OX1 3RE, UK

E-mail: thomas.armstrong@physics.ox.ac.uk

\section{Jamie Graham}

Department of Physics

Durham University

South Road, Durham

DH1 3LE, UK

The extragalactic background light (EBL) is a fundamental cosmological observable of our universe, allowing insight into the history of star formation within our universe. Extending between $0.1-1000 \mu \mathrm{m}$, it is the UV to near-IR that is of interest in high and very high energy astronomy, where EBL photons interact via pair production to leave a visible imprint in the spectra of distant AGN. Multiple studies have been carried out using ground-based Cherenkov telescopes, which can observe the spectra of relatively nearby AGN to provide limits on the density of the EBL. These however do not reveal a great amount of detail concerning the evolution of the EBL with time, and therefore the star formation rate. The Fermi-LAT instrument, with its long exposure of the extragalactic sky, holds an extensive sample of AGN extending out to large redshifts $(\mathrm{z}<2.56)$ and has been used by the Fermi-LAT collaboration to study the EBL. Here we further that study by combining a sample of 259 AGN, carefully modelling their spectral energy distributions and determine a redshift-dependent EBL correction factors to a range of models, taking into account the temporal and spectral variability of sources.

7th Fermi Symposium 2017

15-20 October 2017

Garmisch-Partenkirchen, Germany

\footnotetext{
${ }^{*}$ Speaker.
} 


\section{Introduction}

While there is a certain level of background light encompassing the entire EM spectrum, the term extragalactic background light (EBL) has come to represent background emission from the UV to the far-IR. Like the cosmic microwave background (CMB), which is roughly and order of magnitude larger in intensity and provides data from the earliest epoch of the Universe, the EBL also contains within it important cosmological information. With its two humped spectral shape, the generalised picture is that the UV-optical light measured at earth contains within it the emitted light from stars and galaxies through out cosmic time, with a given fraction of this absorbed by dust and re-emitted in the IR. Therefore measurements of this background can reveal information about the galaxy and star formation history of the Universe [8].

Observations to measure the local intensity of the EBL have been attempted. Firstly through direct measurements, which are often interpreted as upper limits due to foreground contamination. Secondly, integrated galaxy counts which lead to a lower limit due to the assumed incompleteness of the galaxy surveys. See [12] for a review. These measurements, along with an understanding of the comic evolution of the EBL, are important for extragalactic observations with high energy instruments, such as the Fermi-LAT. As the gamma-rays which are observed by these instruments traverse the Universe, they pass through the radiation field of the EBL. If one considers the cross section for $\gamma-\gamma$ pair production, it can be found that for a photon with wavelength of the order of $\mu \mathrm{m}$, that the threshold of interaction leads to a minimum energy of the interacting photon in the range of GeV-TeV. Therefore, for extragalactic sources, the universe starts become opaque to gamma-rays. The optical depth can be quantified as

$$
\tau\left(E_{\gamma}, z\right)=\frac{1}{2} \int_{0}^{z} d z \frac{d l}{d z} \int_{-1}^{1} d u(1-u) \int_{E_{\min }}^{\mathrm{inf}} d E_{b g} n\left(E_{b g}, z\right) \sigma\left(E_{\gamma}, E_{b g}, \theta\right)
$$

where $d l / d z$ is the cosmological line element, $(1-u)$ is the angular distribution, $\sigma\left(E_{\gamma}, E_{b g}, \theta\right)$ is the cross section for pair production and $n\left(E_{b g}, z\right)$ is the number density of the EBL photons.

In order to further understand the EBL, a large amount of work has been put into creating models that reproduce the local intensity, along with providing information about the cosmological evolution, essentially modeling $n\left(E_{b g}, z\right)$ in eqn. 1.1. Based on the methodology, there are often categorised into the following: Forward Evolution (FE) - where the spectral properties of galaxies and stars are obtained by evolving them from cosmological initial conditions [9, 15], Backward Evolution (BE) - where the spectral properties of galaxies and stars are obtained by backward extrapolation of the local galaxy population [7, 10], and Semi-Analytical Models (SAMs) - more elaborate FE models, which include models of galaxy formation and interaction [11, 14]. We note that this does not cover all possible models, just a subset of those commonly used which we investigate in this work. For a review of model types, and the EBL, see [8].

\section{Initial Method}

The aim of this work, was to further that performed in [5] in which a sample of 16 high redshift AGN $(0.85 \lesssim z \lesssim 1.6)$ were used to probe the intensity of the EBL. The method used was the same as that developed in [2] and [13] in which the spectra of high energy AGN were modeled, including the attenuation caused by the energy and redshift dependent EBL optical depth in eqn. 1.1 to give 


$$
\frac{d N}{d E_{o b s}}=\frac{d N}{d E_{i n t}} e^{-a_{E B L} \tau(E, z)}
$$

where $d N / d E_{o b s}$ is the observed flux, $d N / d E_{\text {int }}$ is the intrinsic/unabsorbed flux, $\tau(E, z)$ is the optical depth and $a_{E B L}$ is a correction factor for the optical depth which we derive from in order to place constraints on EBL models. The exponential term results in an observable cutoff in the AGN spectrum, which for all redshifts effects $E_{\gamma} \gtrsim 10 \mathrm{GeV}$. As Fermi-LAT has an energy range of $\sim 10 \mathrm{MeV}$ to over $500 \mathrm{GeV}$, this gives us the unique opportunity to observe both the intrinsic and absorbed parts of the source spectrum. The work in [5] used this to derive the scaling factor for the optical depth $a_{E B L}$, however there were several drawbacks: The effect of source variability was not fully investigated; the intrinsic spectrum was fixed in the EBL fitting step; and there was no determination of systematics from the analysis. We aim to investigate these, along with making further improvements here and in future work.

\section{Updated Method}

Source Selection: In order to obtain more constraining measurements of the EBL, we greatly extended our source selection. Starting with all extragalactic sources $\left(|b|>10^{\circ}\right)$ within the 3FGL [1], which had a detection significance of greater than $20 \sigma$, we looked for associated measured redshift values using the NASA/IPAC Extragalactic Database (NED) and Simbad. This left a total of 259 sources, consisting of 95 BL Lacs, 147 FSRQs and 17 other source classes, covering a redshift range from $0<z<2.5$.

Data Selection: We took 8 years (MET 239557417 to 49232887) of Fermi-LAT Pass 8 reprocessed data between the energy range $100 \mathrm{MeV}<E<500 \mathrm{GeV}$, selecting only FRONT+BACK converting SOURCE class events. The data was filtered using (DATA_QUAL $>0) \& \&($ LAT_CONFIG==1) to ensure LAT was in data-taking mode and to remove sub optimal data. To avoid contamination from the Earth limb a $90^{\circ}$ zenith cut was applied. The Fermi-LAT science tools (v10 r 0 p 5), along with the P8R2_SOURCE_V6 instrument response functions were used for all of our data reduction and initial analysis.

Fitting Method: For each of the 259 sources we first obtain a overall global fit by selecting a $10^{\circ} \times 10^{\circ}$ square region of interest centered around the source and perform a binned likelihood analysis using 10 bins per decade of energy. The model we construct includes the background models provided by the Fermi-LAT collaboration for the galactic diffuse ( $g l 1 \_i e m \_v 06$.fit) and isotropic background (iso_P8R2_SOURCE_V6_v06.txt), along with sources from the 3 FGL catalogue within a radius of $20^{\circ}$. For the likelihood fit, we use the PYTHON package fermipy where we first fit the source of interest individually before freeing all sources with a significance greater than $5 \sigma$ for a final fit.

From the global fit, we extract the source spectral energy distribution (SED), obtaining a likelihood profile for each energy bin. This is done by scanning over the normalisation parameter of a power law with spectral index 2 to represent the flux (where we assume there is minimal spectral curvature within the bin) and calculating the likelihood of the ROI model for each normalisation. Using this likelihood profile SED, we are then able to fit our spectral model including attenuation by the EBL for each model. We assume the model to take the form of a attenuated log parabola, 
i.e. $d N / d E_{\text {int }}$ in equation 2.1 is replaced with $N\left(E / E_{0}\right)^{-(\alpha+\log (\beta))}$ where $N$ represents the overall normalization of the model, $\alpha$ and $\beta$ represent the spectral index and curvature, and $E_{0}$ is the scale energy. By performing this fit outside the Fermi Science Tools, we are able to analyse the whole data set for all 6 EBL models in a fraction of the computational time. We do note that this means that this does not feed back into the global fit for the ROI, potentially leading to systematics. However, the different EBL models that we are testing affect energies greater than $\sim 10 \mathrm{GeV}$, where the LAT point spread function is at its smallest. It is therefore unlikely that the highest energy photons that contribute to the SED would be associated with other point sources and so we are confident that the global fit would not be substantially altered. To check this assumption, we performed two tests, firstly we took a sample of our sources and performed a fit using the Fermi Science Tools along with our method to compare the parameters that the two produced (see Figure 1). The second test we simulated a selection of ROI using the Fermi Science Tool GTOBSSIM, where we then used our method to reproduce the input simulated spectral parameters (including the EBL correction factor). Except for very faint sources, we were able to reproduce the input values within errors.
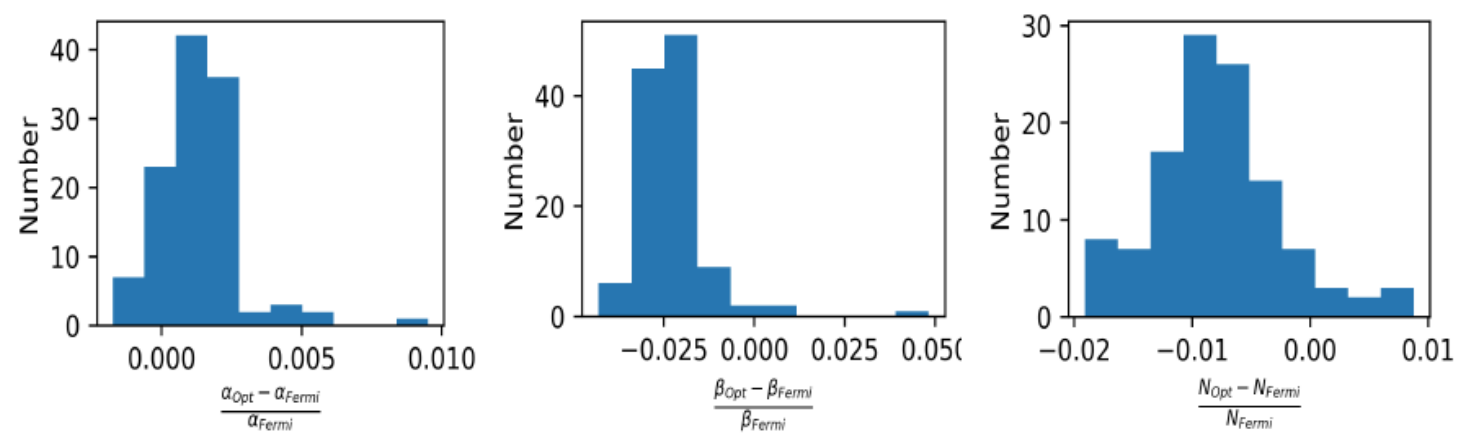

Figure 1: Comparison of the fit parameters obtained with our fitting method $x_{O p t}$ and the Fermi Science Tools $x_{\text {Fermi }}$ where $x$ is the log parabola spectral parameters. It can be seen that the parameters are within $2 \%$, and while there is some small bias, it is negligible in $\Delta \log \mathscr{L}$.

Variability: In 2018, Fermi will celebrate its 10th year since launch. Due to the small effective area of the LAT instrument, it is only through this continuous operation that we have been able to obtain deep observations of these distant AGN, and therefore significant EBL detections. However, it is well known that the majority of these objects are variable, with periods of increased activity and often intense flares. Therefore the SED that we use to model the EBL are a convolution of many different spectral states. We aim to study this further to understand the possible systematics that are introduced by using a time average spectrum. Within the Fermi-LAT catalogues, the definition for variability which is obtained through a likelihood fitting method over $N$ time bins is given by

$$
\left.\mathrm{TS}_{\mathrm{Var}}=2 \sum_{i}^{N}\left[\log \left(\mathscr{L}_{i}\left(F\left(N_{i}\right)\right)\right)\right)-\log \left(\mathscr{L}_{i}\left(F_{\text {const }}\right)\right)\right]
$$

where $\mathscr{L}_{i}\left(F\left(N_{i}\right)\right)$ is the likelihood at the best fit flux in the $i^{t h}$ time bin and $\mathscr{L}_{i}\left(F_{\text {const }}\right)$ is the likelihood of the optimal model for the time averaged fit (assumed constant flux). We therefore create lightcurves for each source, using 97 bins over the 8 years. In addition to this, as our main concern is the effect of the combined spectral states, we define a measure for the spectral variability 


$$
\left.\mathrm{TS}_{\text {spec var }}=2 \sum_{i}^{N}\left[\log \left(\mathscr{L}_{i}\left(F\left(N_{i}, \vec{\theta}_{i}\right)\right)\right)\right)-\log \left(\mathscr{L}_{i}\left(F\left(N_{i}\right)\right)\right)\right]
$$

where now we introduce $\vec{\theta}_{i}$ which represents the set of optimised spectral shape parameters. $\mathscr{L}_{i}\left(F\left(N_{i}\right)\right)$ is the same as defined above, and $\mathscr{L}_{i}\left(F\left(N_{i}, \vec{\theta}_{i}\right)\right)$ is the likelihood for the case where

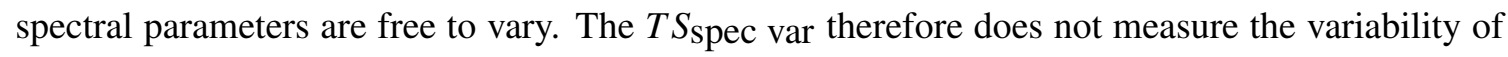
total flux, but rather just the change in the spectral shape. In section 4 we apply preliminary cuts on these parameters, however full evaluation is still required.

\section{Preliminary Results, Conclusion and Further Work}
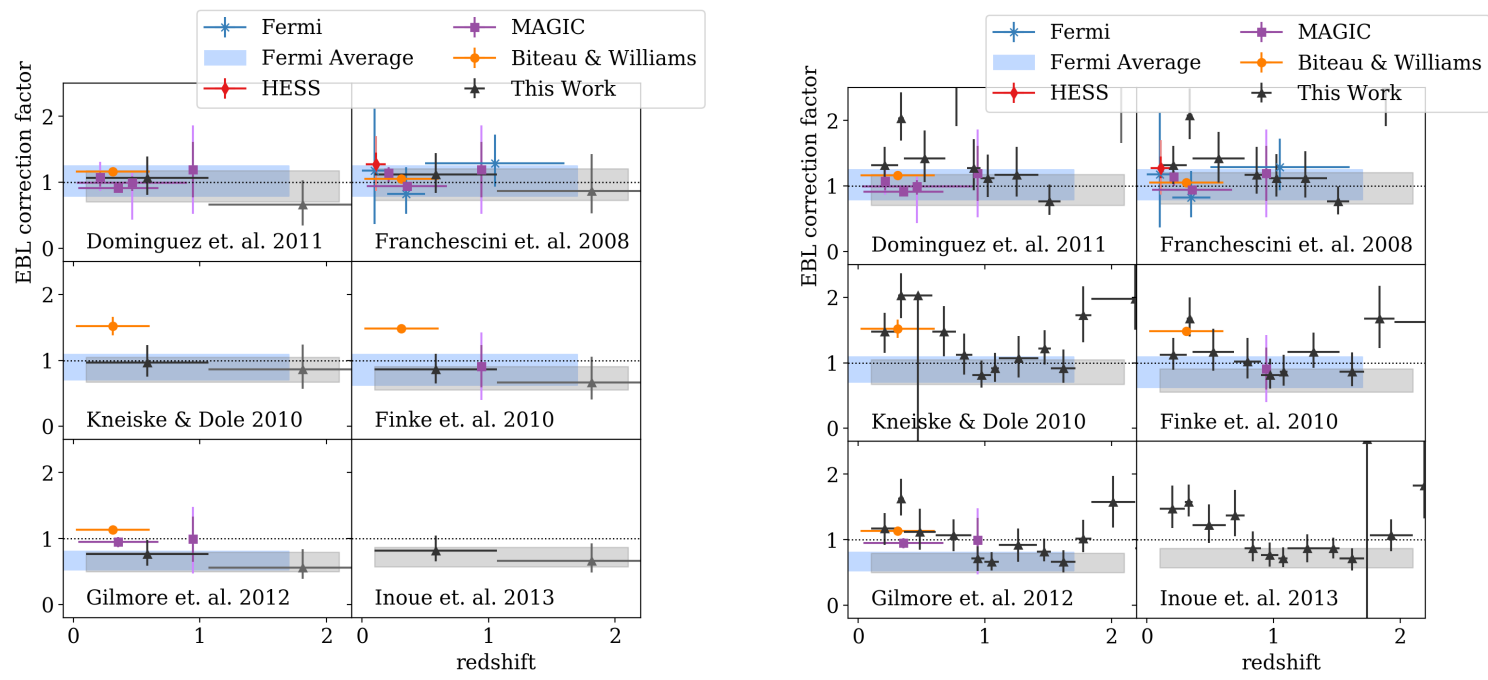

Figure 2: Left: Derived redshift dependent EBL correction factor, including a cut on temporal and spectral variability equivalent to only including sources with $\sigma_{\mathrm{var}}$ and $\sigma_{\mathrm{spec}} \mathrm{var}<8$. The gray band is average over all redshifts, the first black low redshift point has a significance of greater than $5 \sigma$. Right: Derived redshift dependent EBL correction factor without any cuts on variability, showing that there are some points that are effected by outlying sources. The gray band is the average value derived while including the variability cuts. The other points: HESS [13], MAGIC [3], [4] and [16], Fermi-LAT [2] and combined [6]

In this work we have performed a full likelihood and variability analysis on a selection of the brightest 3FGL AGN with associated redshifts, a total of 259 sources. We model each source with a log parabola and include the attenuation from the EBL predicted by the models of $[10,9,15$, $11,7,14]$ to derive a redshift dependent correction factor the the level of attenuation. Preliminary results can be seen in Fig 2 for the case where a cut on the significance of the variability ( $\sigma_{\text {var }}$ and $\sigma_{\text {spec }}$ var $<8$ ) has been applied. Currently the cuts result in a loss of $\sim 90 \%$ of our sources and therefore more careful consideration needs to be placed on the level and combination of these cuts. We also show the case where variability is ignored, and while this results in more redshift bins where the is a significant EBL detection, it can be seen that there is evidently a fraction of sources that have outlying signals. Due to the often difficult task of measuring the redshift for some AGN, 
we will also look into the possibility of errors in published measurements, along with our study of variability in future work.

\section{References}

[1] F. Acero, et al. , Fermi Large Area Telescope Third Source Catalog, APJS, 218, 23, (2015), arXiv:1501.02003 [astro-ph.HE]

[2] M. Ackermann, et al., The Imprint of The Extragalactic Background Light in the Gamma-Ray Spectra of Blazars, Science, 338, 1190, (2012), arXiv:1211.1671 [astro-ph.CO]

[3] M. L. Ahnen, et al, MAGIC observations of the February 2014 flare of 1ES 1011+496 and ensuing constraint of the EBL density, A\&A, 590, A24, (2016), arXiv:1602.05239 [astro-ph.HE]

[4] M. L. Ahnen, et al., Detection of very high energy gamma-ray emission from the gravitationally-lensed blazar QSO B0218+357 with the MAGIC telescopes, A\&A, 595, A98, (2016), arXiv:1609.01095 [astro-ph.HE]

[5] T. Armstrong, A. M. Brown, \& P. M. Chadwick, Fermi-LAT high z AGN and the Extragalactic Background Light, MNRAS, 470, 4089, (2017), arXiv:1705.11185 [astro-ph.HE]

[6] J. Biteau, \& D. A. Williams, The extragalactic background light, the Hubble constant, and anomalies: conclusions from 20 years of TeV gamma-ray observations, APJ, 812, 60, (2015), arXiv:1502.04166 [astro-ph.CO]

[7] A. Domínguez, et al., Extragalactic background light inferred from AEGIS galaxy SED-type fractions, MNRAS, 410, 2556, (2011), arXiv:1103.4534 [astro-ph.CO]

[8] E. Dwek, \& F. Krennrich, The extragalactic background light and the gamma-ray opacity of the universe, Astropart. Phys, 43, 112, (2013), arXiv:1209.4661 [astro-ph.CO]

[9] J. D. Finke, S. Razzaque, \& C. D. Dermer, Modeling the Extragalactic Background Light from Stars and Dust, APJ, 712, 238, (2010), arXiv:0905.1115 [astro-ph.HE]

[10] A. Franceschini, G. Rodighiero, \& M. Vaccari, The extragalactic optical-infrared background radiations, their time evolution and the cosmic photon-photon opacity, A\&A, 487, 837, (2008), arXiv:0805.1841 [astro-ph]

[11] R. C. Gilmore, R. S. Somerville, J. R. Primack, \& A. Domínguez, Semi-analytic modeling of the EBL and consequences for extragalactic gamma-ray spectra, MNRAS, 422, 3189, (2012), arXiv:1104.0671 [astro-ph.CO]

[12] M. G. Hauser, \& E. Dwek, The Cosmic Infrared Background: Measurements and Implications, Annu. Rev. of Astron. and Astrophys., 39, 249, (2011), [arXiv:astro-ph/0105539]

[13] H.E.S.S. Collaboration, Measurement of the extragalactic background light imprint on the spectra of the brightest blazars observed with H.E.S.S., A\&A, 550, A4, (2013), arXiv:1212.3409 [astro-ph.HE]

[14] Y. Inoue, et al., Extragalactic Background Light from Hierarchical Galaxy Formation: Gamma-ray Attenuation up to the Epoch of Cosmic Reionization and the First Stars, APJ, 768, 197, (2013), arXiv:1212.1683 [astro-ph.CO]

[15] T. M. Kneiske, \& H. Dole A Lower-Limit Flux for the Extragalactic Background Light, A\&A, 515, A19, (2010), arXiv:1001.2132 [astro-ph.CO]

[16] D. Mazin, et al., EBL Constraints Using a Sample of TeV Gamma-Ray Emitters Measured with the MAGIC Telescopes, AIP, 1792, 050037, (2017), arXiv:1610.09633 [astro-ph.HE] 